\title{
Coreografie dantesche
}

\author{
Francesco Ciabattoni
}

Georgetown University

fc237@georgetown.edu

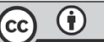

\begin{abstract}
Le più notevoli scene di ballo nella Commedia hanno come protagoniste delle donne o degli uomini paragonati a donne. Dalla danza delle virtù teologali nel paradiso terrestre alla danza dei teologi nel Cielo del Sole e a quella dei santi Pietro, Giovanni e Giacomo, Dante usa la figura femminile come paragone della grazia coreutica, sfidando una lunga tradizione che inibiva il ballo muliebre. Questo saggio propone di inquadrare l'arditezza rappresentativa di tali scene nella cornice della tradizione teologica e della lirica cortese e francescana.
\end{abstract}

Parole chiave: Dante; Commedia; danza; ballo; musica; poesia medievale; lirica medievale; arti performative.

\begin{abstract}
The most striking scenes of dancing portrayed in Dante's Commedia feature either female protagonists or male characters likened to women in a simile. From the dance of the theological virtues in the earthly paradise to the dance of the theologians in the Heaven of the Sun, as well as the dance of saints Peter, John and James, Dante uses feminine figures as a paragon of choreographic grace, challenging the long-standing tradition that forbade female dancing. This essay proposes to frame the representational boldness of such scenes within the tradition of theological scholarship on one hand, and that of Franciscan devotion and court lyric on the other.
\end{abstract}

Keywords: Dante; Comedy; dance; ballet; music; medieval poetry; medieval lyric; performing arts. 
"Largior hic campos aether et lumine vestit purpureo solemque suum sua sidera norunt pars in gramineis exercent membra palaestris contendunt ludo et fulva luctantur harena pars pedibus plaudunt choreas et carmina dicunt nec non Threicius longa cum veste sacerdos obloquitur numeris septem discrimina vocum." (Aen. VI, 640-644)

e nell'Eneide la raffigurazione virgiliana degli eroi, dei sacerdoti e dei poeti $\checkmark$ che danzano e cantano inni in onore di Apollo nei Campi Elisi non poneva difficoltà di tipo teologico o morale, l'analoga scena dantesca della danza dei teologi nel Cielo del Sole va invece inquadrata entro i parametri delle tradizioni teologica, morale e lirica senza le quali non se ne può comprendere l'ardito sincretismo artistico. Com'è noto, la tradizione religiosa del Medioevo ebbe reazioni alquanto diverse, talvolta di profonda diffidenza, nei confronti della danza: invenzione diabolica e pratica sconveniente, più adatta ai giullari e agli istrioni che al buon cristiano, la danza fu spesso severamente regolata o addirittura censurata dalle autorità ecclesiastiche sin dal quarto secolo quando gli Atti di Giovanni - in cui si narrava che Gesù danzasse con gli apostoli la notte prima di essere crocifisso - vennero esclusi dal canone biblico. Uno studio di Sandra Pietrini (2000) ricapitola efficacemente il biasimo che gli scrittori cristiani ebbero per l'espressione del movimento corporeo. Tuttavia, agli esempi negativi di danza (il ballo di Salomé, $M t$. I4:6, Mc. 6:22; la danza degli israeliti davanti al vitello doro Es. 32:19) le Scritture affiancano anche esempi positivi (la gioiosa danza di David davanti all'arca dell'alleanza, II $S a$ muele 6:I4-I6; il ballo di Myriam e delle donne di Israele, Es. I5:2I), il che ha permesso il parallelo sviluppo di un atteggiamento più aperto nei confronti della danza, di cui si dirà sotto.

Il monito di teologi e moralizzatori circa la danza ha radici antiche (già Agostino commentava negativamente l'usanza di ballare durante il sabato: "melius enim utique tota die foderent, quam tota die saltarent" Enarrationes in Psalmos, XXXII.ii.6) e durava ancora in Italia ai tempi di Dante. Se la tradizione dei balli di corte venne poi progressivamente rivalutata, come si può apprezzare nel Decameron, nei trattati De arte saltandi et choreas ducendi di Domenico da Piacenza (ca. I450) e De pratica seu arte tripudii vulgare opusculum di Guglielmo Ebreo da Pesaro (1463), fino alla consacrazione artistica nel tardo Cinquecento nell' Orchésographie di Thoinot Arbeau, tuttavia frati e predicatori mantennero un atteggiamento severo specialmente nella Firenze dell'epoca di Dante. Per esempio, Domenico Cavalca ammoniva che "le vanità e le pompe vane [...] sono ne' balli sempre opera del diavolo; e questo si mostra in ciò che sempre ne' balli si procede da mano manca, 
della quale, come dice il Vangelo, stanno i dannati" (Cavalca I837: cap. XVIII, 252), mentre Stefano di Borbone e fra Giordano da Rivalto (anche conosciuto come Giordano da Pisa, I260-I3II) ribadivano l'attribuzione dell'invenzione della danza al demonio. ${ }^{\mathrm{I}}$ Negli ambienti religiosi prevalse dunque, ai tempi di Dante, un atteggiamento di generale diffidenza, specialmente da parte di religiosi e predicatori, per i quali le forme di devozione popolare che prevedevano il movimento dei corpi sapevano pericolosamente di rito pagano e dissolutezza morale.

Non si debbono però confondere le forme di danza sacra, pure ampiamente diffuse nella devozione popolare di tutta Europa (Horowitz 1989: 288), con la liturgia propriamente detta (Anglès 1975: 35I), che fu regolata alquanto severamente con l'obiettivo della sobrietà. Sono molti i documenti, specialmente in Francia, in cui si interdicono nelle chiese choreae, saltationes e ballationes perché ritenute oscene (Gougaud 9-I3). Fra queste spicca un divieto di Petrus Bertrandus, vescovo di Autun, che nel 1323 si trovava a dover espressamente vietare le choreae nelle chiese, nei cimiteri e nelle processioni: "nec in ipsis ecclesiis vel cimiteriis praesumant aliqui choreas facere, vel dicere cantilenas" (Martène I7I7: col. 50I, XVII). Tali divieti, che si reiterano da un secolo all'altro (Haines 20I0: I62-17I), spesso riguardano precipuamente le donne, alle quali erano interdetti sia il canto che il ballo, come si legge per esempio in questo Concilio romano dell'826:

Sunt quidam et maxime mulieres, qui festis ac sacris diebus atque sanctorum nataliciis non pro eorum quibus debent delectantur desideriis advenire, sed ballando et verba turpia decantando, choros tenendo ac ducendo, similtudine paganorum peragendo, advenire procurant. (Werminghoff 1908 : 58I)

Ma proprio la ripetizione dei divieti in numerosi statuti e concili indica al contrario la persistenza di tali forme devozionali. E poiché il canto e il ballo, e soprattutto delle donne, erano manifestazioni sia religiose che secolari più diffuse di quanto la storiografia medievale abbia sinora riconosciuto (Haines 20IO: 5I e segg.), in questo mio contributo propongo di guardare agli episodi coreutici nella Commedia adottando non solo il punto di vista della tradizione teologica, ma anche quello della lirica d'amore e della poesia devozionale francescana. Da tale specola si potrà meglio far luce sulle scene di danza specie nel Purgatorio e nel Paradiso, poiché esse presentano l'enigma della connotazione ambivalente del genere dei danzanti. Infatti nelle scene più teologicamente significative sono proprio figure femminili a danzare, o figure maschili paragonate a donne, da Matelda paragonata a "donna che balli, e piede innanzi

I. Stefano di Borbone: "Diabolus est choreizancium et canciarum inventor" (I877: 397); Giordano da Rivalto: "Vedi altresì come sono male cose questi balli e questi giuochi [...] Opera è di Demonio" (I839: 227). Cfr. Padovan 1985: 5; S. Pietrini 2000; e Buttà 20I4: passim ma specialmente II3. 
piede a pena mette" (Pg. XXVIII 52-54), alla vera e propria danza delle virtù cardinali e teologali ( $P g$. XXXI I04; $P g$. XXIX I2I-I22), fino alle ardite similitudini femminili per i santi del paradiso.

Un esempio tratto appunto dalla terza cantica, il moto in cerchio delle anime del Cielo del Sole ( $P d$. X 76-81; Pd. XII 22), assume un valore metafisico che esprime la perfezione geometrica e ontologica dell'universo tutto: non solo, com'è evidente, l'universo è strutturato secondo un principio geometrico tridimensionale e sferico (si chiamano per l'appunto sfere celesti, anzi "spere", per dirla con Dante), ma la stessa visione di Dio al termine ultimo del viaggio dantesco consiste nell'impenetrabile forma di tre circoli (ma Dante dice "giri", il che suggerisce il movimento circolare, $P d$. XXXIII II7) di tre colori diversi ma d'una stessa dimensione che spirano (v. I2O) in una circolazione (v. I27) perfettamente sincronizzata. Ed è proprio questo rapporto geometrico dell' "imago al cerchio" (v. 138) che sfugge alla ragione del pellegrino finché, folgorato dall'illuminazione divina, egli pure, nella perfetta armonia interiore di desiderio e volontà, inizia a girare "sì come rota ch'igualmente è mossa" (v. I44). Dante ricorre a modelli di movimento sincronico con una metaforologia che investe altri campi: orologeria, musica, fisica e geometria, senza condannare la forma coreografica perché "una delegittimazione senz'appello avrebbe compromesso [...] la versatilità semantica del ballo, estromettendolo dal repertorio figurale 'alto'” (Fratini 20I4: 87). Si può partire, dunque, dall'audace similitudine in cui Dante paragona l'apostolo Giovanni a una vergine lieta che si unisce al ballo di Pietro e Giacomo:

E come surge e va ed entra in ballo vergine lieta, sol per fare onore alla novizia, non per alcun fallo, così vid'io lo schiarato splendore venire a' due che si volgieno a nota qual conveniensi al loro ardente amore.

Misesi lì nel canto e ne la rota; e la mia donna in lor tenea l'aspetto pur come sposa tacita ed immota. (Pd. XXV, IO3-III)

L'enigma delle donne che ballano, oltre a comparire qui sopra, pare farsi anche più fitto quando si considerino le similitudini che vedono i ventiquattro spiriti sapienti del Cielo del Sole come "donne non da ballo sciolte" ( $P d$. X 79) poiché esse sfidano apertamente la proibizione che le autorità ecclesiastiche decretavano con particolare severità per il ballo femminile: Prudenzio parla della lussuria come di una "saltatrix ebria" (Psicomachia, v. 380), mentre Giovanni Crisostomo associa senza mezzi termini la danza di Salomè (che non nomina neppure) con il diavolo: "ubi enim saltatio ibi diabolus" (Homiliae 
in Matthaeum, PG 58:49I.3). Il manoscritto IoAir del Museo MeermannoWestrenianum all'Aia, che contiene il De civitate dei di Agostino, emblematizza la città infernale con un gruppo di due donne che ballano nude insieme a due giullari e un diavolo (Pietrini 20Io), poiché il ballo delle donne era percepito come particolarmente scandaloso. Il già ricordato Stefano di Borbone apre la sua invettiva contro la danza proprio ammonendo che "timenda sunt mulierum consorcia ... maxime autem declinanda sunt loca in quibus fiunt choree, et ipse choree fugiende" (I877: 397), quindi riferisce l'aneddoto di un sant'uomo indotto al peccato da una danzatrice e conclude che l'inventore di tali pratiche non può che essere Satana. Allo stesso modo, il Salterio de la reina Maria (British Library Royal 2 B VII, f. I66) contiene immagini di una donna che balla col diavolo (Buttà 20I4: II3). E si potrebbe continuare con Guglielmo Peraldo, Umberto di Romans, Tommaso di Cantimpré e altri (Page 1989: IIo e segg.) che si accanivano contro il ballo, in particolare se esso coinvolgeva le donne.

Questi ripetuti divieti e ammonizioni tradiscono, però, una realtà alquanto diversa: se si sentiva il bisogno ricorrente di porre un freno alle attività coreutiche, esse dovevano evidentemente essere piuttosto frequenti malgrado l'aperta riprovazione delle istituzioni. Ne sono prova inni come "Jesu corona virginum", anticamente attribuito ad Ambrogio, in cui Gesù è accompagnato da vergini danzanti ("Qui pascis inter lilia, Septus choreis virginum, Sponsas decorans gloria"; Gougaud 19I4: 7). La questione appare dunque complessa e non si possono ignorare i testi, sia religiosi che profani, in cui la danza ha invece una funzione celebrativa della gioia di vivere, ma è anzi da qui che si possono prendere le mosse per spiegare il ricorso dantesco alla similitudine dei beati con donne danzanti.

Infatti, una tradizione intellettuale che riconosceva alla danza una valenza positiva persistette per tutto il Medioevo, come ha magistralmente illustrato John Freccero (1989). Nei miti platonici dell'anima mundi e della chorea stellarum - poi cristianizzati e confluiti negli Atti di Giovanni e nel Commento di Macrobio al Timeo - lo studioso americano individua i principi regolatori della coreografia trinitaria di $P d$. X, mettendo in relazione la "danza zodiacale" dei sapienti con vari testi astronomici dalla Consolatio philosophiae all'Apocalisse (Freccero 1989: 298 e segg.). Se le forme coreografiche dei sapienti sono "un modo per renderli [i sapienti] prossimi all'esperienza del lettore" (Ardissino 2009: I23), non sembra che la sublimità di questo cielo ne esca sminuita, anzi l'obbiettivo di Dante è piuttosto di avvicinare il lettore alla nobiltà spirituale dei sapienti attraverso una sublimazione delle forme devozionali più popolari, cioè quelle del ballo delle donne.

La macro-sequenza di $P d$. X-XIV è d'altronde stata oggetto di interesse critico con approcci disparati: c'è chi ne ha sottolineato la "coerente organicità 
musicale e liturgica" (Cappuccio 2008: 149), chi ha giustamente ricordato che l'elemento coreografico contribuisce a compensare "l'indicibilità dell'esperienza" (Ardissino 2009:II9). John Secor ha visto una relazione di corrispondenze e rovesciamenti parodici fra la danza dei tre sodomiti fiorentini di If. XVI, la danza delle tre virtù in Pg. XXIX e la danza di Pietro, Giovanni e Giacomo in $P d$. XXV. Altri hanno veduto nella danza dei dottori della chiesa di $P d$. X-XIV una trasposizione sacra del genere profano della ballata, sulla scorta di una segnalazione di Vincenzo Borghini (Catelli 2008: 12I). ${ }^{2}$ Catelli e Cappuccio (2008: 154, 158) propongono di ricondurre le danze dei sapienti di $P d$. $\mathrm{X}$ all'ambiente della lirica volgare di argomento non sacro, e cioè al genere della ballata, pur concedendo che l'esecuzione musicale di Tommaso d'Aquino, Bonaventura e gli altri è di fatto un brano polifonico di natura sacra. E certo, a sostegno di questa lettura si può citare il riferimento all'aubade trobadorica che diventa una versione sacralizzata di canto degli amanti (Ciabattoni 20I0:I64): nel richiamo dell'orologio che chiama la Chiesa a "mattinar", cioè a festeggiare il suo sposo (Dio), si vede la strategia dantesca di riscrivere in funzione religiosa le forme della poesia profana:

Indi come orologio che ne chiami ne l'ora che la sposa di dio surge a mattinar lo sposo perché l'ami. (Pd. X, I39-I4I)

La danza dei sapienti ha origini antiche, a partire dalla danza salica pagana (Anglès 1975: 352; Cirilli I9I3: 30 segg.) fino alle choreae e ai tripudia che, sfidando le proibizioni, talvolta accompagnavano i canti sacri nelle chiese del tardo Medioevo. Il costume di far danze nei luoghi di culto con funzione celebrativa e ricreativa persisteva malgrado tutto in diverse zone d'Europa: Yvonne Rokseth mostra come a Notre Dame ai giovani si concedessero momenti ricreativi con balli e canti in chiesa, specialmente durante le lunghe celebrazioni di Natale e Pasqua, e ci informa che a Parigi si derogava dal divieto di ballare in chiesa proprio per evitare che i giovani cadessero in tentazioni; quindi la gioventù clericale parigina del tredicesimo secolo "sautillait en cadence, en chantant des morceaux latins" (Rokseth 1947: 94), mischiando alla danza canti in latino. Il Cartulaire de l'église de Notre Dame de Paris (Guérard I850: 4I6) attesta che queste manifestazioni extra liturgiche erano tollerate specialmente nei giorni di Santa Caterina e San Nicola. Ciò accadeva in accordo con il pensiero di teologi influenti - e collocati da Dante fra i danzatori in $P d$. X - come

2. Fra questi Sapegno, nel suo commento alla Commedia (Alighieri 1968: PdX. 79); Nardi 1956: 650 segg.; e Lograsso 1965, poi seguiti da Catelli 2008. Il commento di Vincenzo Borghini (I897: I8O-I8I), a lungo inedito, si trova stampato nel "Bullettino della Società Dantesca" dove si legge che è stato preso dalla p. 38 del manoscritto Bigazzi 42 della Biblioteca Moreniana di Firenze. 
Alberto Magno, che ammetteva l'utilità sociale della danza, della musica strumentale e dei generi teatrali come sfoghi emotivi:

Delectationes autem quidem sicut cantoria, viellatoria, tripudia, saltatoria, tragoedia, et comoedia, et hujusmodi, quas oportet politicum sufficienter praeordinare, ut omnis civis jucundus apud civem in delectabilibus liberalibus inveniatur. (Alberto Magno I897: Lib.I, tract.3, cap.I, p.30b) ${ }^{3}$

E sulla scia di Alberto, Bonaventura analogamente assolveva la danza in sé, rifacendosi all'episodio di Myriam nell'Esodo, e condannandone piuttosto i modi quando erano lascivi e il fine quando era provocatorio:

Dico igitur, quod ludus chorearum non esto malus secundum se, sicut pater de sorore Moysi, Exodi decimo quinto, sed fit malus quadruplici de cause scilicet propter modum, quando est modus lascivus; protper finem quando fit ad provocandum libidinem ; propter tempus, ut non fiat tempore tristitiae; propter personam, u non fiat a persona religiosa... His enim quatuor de causis possunt male fieri et fiunt; his exclusis bene possunt fieri. (Bonaventura de Bagnorea I889: Tomus IV, Distinctio XVI, Dubitatio xiii, pp. 4OI-402)

Le danze circolari dei religiosi si distinguevano da quelle della gente comune, più vicine alle manifestazioni buffonesche degli istrioni di strada, ancorché nei documenti del dodicesimo e tredicesimo secolo il termine latino più usato per tali balli fosse tripudium (Rokseth 1947: 95-97), lo stesso impiegato da Dante in $P d$. XII 22. Fra le numerose miniature nei manoscritti che rappresentano chierici danzanti, una delle più note è a f. $428 \mathrm{r}$ del famoso Magnus Liber Organi (Firenze, Biblioteca Medicea Laurenziana, Plut. 29.I, un manoscritto prodotto a Parigi intorno al I250 e appartenuto a Piero de Medici dal I456 (Fig. I).

La figura I mostra cinque ecclesiastici, uno parrebbe un frate francescano dal saio, che si tengono per mano guardandosi negli occhi, il che ha permesso ai musicologi di affermare che la sezione di mottetti sacri che seguono erano intesi per essere cantati e danzati (McGee 1989: 6; Rokseth 1947: I06). È significativo che la miniatura dei monaci che danzano sia stata usata per decorare un codice così elegante che contiene centinaia di mottetti polifonici della scuola di Notre Dame. Il ventottesimo volume degli Analectica Hymnica Medi Aevi raccolti da Blume e Dreves (1898), dedicato agli uffici liturgici in rima, raccoglie parecchi testi latini che fanno riferimento alla danza accompagnata dal canto sacro. ${ }^{4}$ Se dunque si può accogliere la proposta di Catelli (2008: 132)

3. E si veda anche l'opinione di Tommaso d'Aquino (ST, IIa IIae, I68) che alla domanda "utrum possit esse aliqua virtus circa actiones ludi" risponde citando Aristotele (Ethica Nicomachea II) che "motus corporales exteriores sunt hominibus a natura [...] ego circa tales motus non attenditur aliqua virtus".

4. "Psallat cum tripudio Caeli sumpta solio" (98); "In organis et citharis Cunctis devictis liostibus Et mentium tripudiis Cum dilatatis cordibus" (I44); "Commune fit tripudium, Continuum vocis Organum (I67); "Celebri laudum iubilo Persolvamus odas Christo ... Triumphale tripudium" (I75); "Cum sanctorum tripudio ; Laus uni trino Domino" (209); 
che i sapienti del cielo del sole si fanno umili nella danza - proprio come, nel parallelo canto X del Purgatorio David si era fatto "trescando, alzato, umile salmista" - si dovrà tenere conto che la danza associata al canto gioioso in paradiso serve un fine soprattutto gratulatorio ed encomiastico, esprimendo la laus dei "con una sorta di possessione individuale e collettiva" (Ardissino 2009: I32) che al contempo non prescinde da una dimensione liturgica e teatrale (Vettori 1997: 83).

Accanto all'elemento teologico andrà però esplorato un altro elemento cui il poeta della Commedia ricorre senza il timore di apparire inopportuno. $\mathrm{Si}$ tratta della tradizione lirica amorosa in volgare, alla quale non erano estranei i toni e le formule della devozione popolare, e numerose sono le menzioni di donne a ballo. Giuseppe Ledda (2015: 236 n.25) segnala per esempio una canzone del fiorentino Francesco Ismera Beccanugi ("Per gran soverchio di dolor mi movo", contenuta nel codice Riccardiano 2846 e nel Chigiano L. VIII. 305) che potrebbe avere a che fare con la danza di David in Pg. XI, per via dell'uso del termine "tresca", che come osserva Ledda serve a illustrare l'umiliazione del salmista attraverso una forma di ballo popolare. Ai fini della presente riflessione, varrà la pena di notare che fra le piacevolezze segnalate da Beccanugi si trovano "donne e donzelle in danza gire a tresca", dunque un ballo femminile in ambiente secolare, di cui si trovano testimonianze anche iconografiche. D'altronde, la dicotomia fra la danzatrice come tentatrice e la danzatrice come esempio di virtù virginale e trascinatrice della fede è archetipo biblico incarnato da Salomé e Myriam (Apostolos-Cappadona 1993: 97). Nella Toscana del Trecento era accettabile raffigurare il ballo casto delle donne se nel I338 Ambrogio Lorenzetti affrescava la Sala dei Nove del Palazzo Pubblico di Siena collocando una danza di donne cantatrici fra gli effetti del buon governo (Fig. 2). 5

Si tratta, come appare evidente, di una scena di socialità urbana e secolare, che non ha nulla di liturgico né di licenzioso, ma accanto alle rappresentazioni pittoriche e letterarie di ricreazioni sociali aristocratiche - progressivamente accettate dagli anni '3o del quattordicesimo secolo - Maurizio Padovan (1985: I5)

"Laudes psallat praeconio Virtutum tripudio, Mater pangat ecclesia Victorini sollemnia" (267).

5. Quentin Skinner (1999:15-16) suggerisce che le figure danzanti siano in realtà maschili perché troppo alte e senza seno. Tuttavia a ben guardare tutte le figure indossano vesti che appaiono femminili. Inoltre, delle figure più alte, quella che regge il tamburello ha seno, mentre altre due, che pure sembrano indossare vesti femminili, sono girate di spalle e dunque non si può affermare che siano senza seno. Le figure più piccole potrebbero essere fanciulle, il che spiegherebbe perché appaiono "flat-chested". La posizione di Skinner, sebbene autorevole, appare in minoranza giacché Rowley, DeWald, Eorsi, Bowsky, Starn e Partridge e Frugoni (si veda la nota 60 a p. I5 dello stesso Skinner) concordano che le figure danzanti di Lorenzetti siano invece femminili. E di quest'ultimo parere sembra essere pure Barbara Sparti, almeno per quanto riguarda la figura che suona il tamburello (Dance, Dancers and Dance-masters in Renaissance and Baroque Italy, Massimiliano Piretti Editore, Bologna, 2015, p. 404). 
segnala anche il "ballo delle donzelle nel giardino d'amore" nell'affresco di Andrea Bonaiuti nel Cappellone degli Spagnoli (Firenze, Santa Maria Novella), un dettaglio della Chiesa militante e trionfante (Fig. 3, I365-I368) e una scena allegoricamente sacralizzata.

Siamo ancora lontani dalle allusioni dantesche al ballo muliebre in contesti sacri: nei passi ricordati del Purgatorio e del Paradiso, l'elemento coreografico risulta esaltato, sublimato e depurato di ogni sensualità proprio perché a danzare sono le virtù teologali e i santi del paradiso, e perché queste performance si svolgono in un luogo e in un contesto da cui ogni impudicizia è per definizione bandita.

Ma l'idea che una danza mistica possa esprimere il fervore religioso sarà stata familiare al poeta della Commedia grazie alla tradizione lirica del misticismo francescano. Una lauda che il ms. Riccardiano III9 (f. 193b) attribuita a Jacopone da Todi, ma probabilmente spuria, esprime la frenesia del fuoco d'amore divino con toni di struggente desiderio (cito il testo da Bartolomeo Sorio I858: IO-II):

Ciascuno amante che ama il signore

Venga a la danza cantando d'amore.

Venga alla danza lieto innamorato

Disiando quello che già l'ha creato,

Di amor ardendo il cor tutto infocato

Sia trasformato di grande fervore.

Infervorato dall'ardente foco,

Come impazzito che non trova loco,

Cristo abbracciando no l'abbracci poco,

$\mathrm{Ma}$ in questo gioco se gli strugga il core.

Lo cor si strugge come al foco il ghiaccio,

Quando col mio Signor dentro mabbraccio;

Gridando amor, d'amor sì mi disfaccio,

Con l'amor giaccio com'ebrio d'amore

Questa retorica amorosa consente di evitare la contaminazione dell'eros, mantenendo però la tensione del desiderio - e addirittura dell'ebbrezza esplicitamente condannata da Prudenzio in associazione al ballo - anche nella sua dimensione fisica, che è tipica della poesia cortese. A questa lauda fa eco un'altra, attribuita a Ugo Panziera, nel ms. I68 della Biblioteca Nazionale Centrale di Firenze (f. 29r), ma altrove attribuita a Jacopone (vedi Zimei 20Io: 334 n.57):

Una rota se fa in cielo con tucti santi in quel giardino, la dove sta l'amor divino che-lli infiamma dell'amore. In quella rota vanno li sancti 
con l'angely tucty quanty,

a quello sposo stanno davanty

et tucty danzano per amore.

In quella rota e un'allegranza

de uno amore desmisuranza,

tucti vanno ad una danza

per amor del Salvatore.

Il riferimento alla rota dei santi e all'eccesso di amore divino, che non può essere espresso a parole, anticipa il tema dantesco della ineffabilità cui sopperiscono musica e danza in $P d$. X.

Il lungo testo di questa lauda / ballata presenta proprio San Pietro e San Giovanni (anzi, entrambi i Giovanni, Battista e Evangelista), se non proprio femminizzati come in Dante in Paradiso XXV, tuttavia ringiovaniti e ingentiliti dal ballo stesso e dalle ghirlande, in virtù della loro capacità di amatori, ovviamente amatori di Cristo e della Chiesa, dunque di un amore intenso e nobilitante (cito da Zimei 2010: 335-6):

Amendue li san Giohannj

son vestiti ad novj tagli,

àndo tante belle gerlande

perché forono grandy amatory

[...]

Chi vedesse sancto Pietro

che pareva che fusse anticho

egli è sì iniovenito

che pare che sia un garzone.

Inoltre la lauda "Regina pretiosa" contenuta nel ms. Banco Rari Palatino i8 della Biblioteca Nazionale Centrale di Firenze rappresenta la divina corte con canti e danze cui prendono parte tutti i santi compresi gli evangelisti (cito da Wilson I995: lxx):

la 've sonno li vangelista e Marco e Luca

et sonnvi tucti i santi

che fanno i dolçi canti

davanti alla regina fanno dança.

Questi precedenti lirici stabiliscono "un suggestivo parallelo fra la realtà spirituale e il mondo cortese attraverso la sublimazione di modelli terreni efficacemente complementari, quali la musica e il ballo" (Zimei 20I0: 335), permettendo a Dante di inserire le proprie raffigurazioni coreutiche nel solco di una tradizione che al contempo le giustifica poeticamente e consente al poeta di superarle con maggiore arte. Pure vicino alla situazione dantesca è il sentimento della pazzia per amore di Cristo, già espressa in "Ciascuno amante" ("infervorato dall'ardente foco / come impazzito") che invita i fedeli a 
danzare per esperire l'eccesso di amore divino ("chi vòle entrare en questa danza / trova amor d'esmesuranza"), un paradosso consentito nell'ambito della stultitia propter Christum che Jacopone declina in "Senno me par e cortesia / empazzir per lo bel Messia". Ciò porta Dante a recuperare l'intensità del desidero e la follia d'amore senza cadere nella fol'amor che aveva indotto Guittone d'Arezzo ad abbandonare la lirica d'amore e a equiparare "amor" e "folor". E tuttavia, il modello più prossimo a Dante è forse rappresentato da due distinte ballate di Guittone, la cui influenza si fa sentire persino nella Commedia (cito da Contini 1960: I 230-23I):

\section{XXXIX}

Vegna, vegna - chi vole giocundare e a la danza se tegna!

[...]

Or venite, venite e giocundate, sponse del mio Signore e donne mie, e de tutt'allegrezza v'allegrate, amando lui de pur cor ciascun die. [...]

Tegna, - tegna, chi cher pene, penare, e a Tua danza non vegna.

\section{L}

Ora vegna a la danza, e con baldanza - danzi a tutte l'ore chi spera in voi, Amore, e di cui lo cor meo disia amanza. Oh, quanto è dilettoso esto danzare in voi laudare, - beata Maria! E che maggior dolcezza e dilettore Ch'aver di voi, Amor [...]

Con il primo testo, "Vegna, vegna" (ballata XXXIX dell'edizione Egidi, Laterza 1940), Guittone invita proprio le donne a unirsi alla danza, con cuore puro, per il Signore. Quanto all'altra ballata, "Ora vegna a la danza", numerata L da Egidi, essa è corredata, nel Codice Palatino $4 \mathrm{I} 8$ (già Banco Rari 2I7) al f. 58v da una miniatura che illustra due donne e due uomini che danzano (Fig. 4).

La partecipazione delle donne a queste danze mistiche non può non far riflettere, se consideriamo il ruolo profondamente formativo che, malgrado la ripetuta e ostentata condanna poetica (De vulg. I xiii I; De vulg. II vi 8; Pg. XXIV e Pg. XXVI), la poesia di Guittone ebbe per Dante. Barbi e Maggini (1956: 40) notavano già - come pure Foster e Boyde (1967: I-7I) e Moleta (I976: 2) - il debito stilistico del primo Dante lirico e della Vita nuova nei confronti di Guittone, ma Teodolinda Barolini (1984: I00-IO6) ha dimostrato 
che l'influenza dell'aretino si esercitò anche nelle rime petrose, nelle canzoni morali e nella Commedia, e ben aldilà delle due menzioni in chiave di superamento critico del Purgatorio. Anzi, proprio in corrispondenza di $P d$. XI e XII si potrebbe vedere un antecedente guittoniano nei panegirici di San Francesco e San Domenico perché pure Guittone scrisse eulogie poetiche per questi santi. Più recentemente Tristan Kay ha parlato apertamente di "Oedipal anxiety" (2016: 96) da parte di Dante verso Guittone, osservando che l'ostilità tradisce e smaschera un debito tematico, strutturale e stilistico. Ma ritorniamo per un momento al suggerimento di Barolini che la lode dei santi in $P d$. XI e XII possa essere influenzata da Guittone. A ben guardare, in effetti, "Maraviglioso beato", la lode di Guittone per Domenico, ha lasciato una traccia tutt'altro che superficiale in $P d$. XII, a cominciare dalla ricorrente metafora dantesca di Domenico come coltivatore dell'orto e della vigna di Cristo, che trova diretta ascendenza in questi versi di Guittone:

Agricola a nostro Signore

non terra ma cor' - coltando

Si noti come Dante ripropone la metafora, che poi governerà l'intero canto, con lo stesso vocabolo:

Domenico fu detto; e io ne parlo sì come de l'agricola che Cristo elesse a l'orto suo per aiutarlo (Pd. XII, 67-72)

E per continuare, Dante estende il discorso onomastico ai genitori del santo, fondandone la veridicità nella coincidenza del significato e del significante:

Oh padre suo veramente Felice! oh madre sua veramente Giovanna, se, interpretata, val come si dice!

(Pd. XII, 79-8I)

I nomi dei genitori di Domenico significano "felice" e "grazia di Dio" (BoscoReggio), e quindi essi sono veramente felice e la grazia di Dio, secondo la pratica etimologica medievale che vuole che "li nomi seguitino le nominate cose" (Vita Nuova XIII 4), e che quindi il significante rifletta in sé l'essenza del significato. La terzina dantesca ricalca da vicino il modulo guittoniano con invocazione del vero significato dato dal nome:

O vero Domenico...

$\mathrm{O}$ nome ben seguitato

e onorato - dal fatto,

Domenico degno nomato,

a Domino dato 
La formula laudatoria di Guittone, dunque, sostiene che il nome di Domenico significhi "dato dal Signore" (a Domino dato), una struttura grammaticale che Dante giustamente corregge perché "Dominicus" significa propriamente "del Signore", un possessivo che indica appartenenza, e non "dato dal Signore" (ablativo: a Domino), con enfasi sulla provenienza come vorrebbe l'aretino:

e perché fosse qual era in costrutto,

quinci si mosse spirito a nomarlo

del possessivo di cui era tutto.

Domenico fu detto...

(Pd. XII, 67-70)

Le somiglianze fra le due lodi non terminano qui; per esempio entrambe mettono in luce positiva il ruolo di Domenico come "paladino" ( $P$ d. XII I42) della chiesa contro "gli sterpi eretici" ( $P d$. XII Ioo), che Guittone esprimeva così: "a la Chiesa tu defensione / e forte campïone / [...] t'ha per socorso noi dato". Dante censore di Guittone, ne riscrive la lode di Domenico, occupando il luogo del "sommo laudatore" invocato dall'aretino che, in una dimostrazione di humilitas retorica, si dichiara forse non degno di esprimere la lode di Domenico:

Forse ch'io perdo tacere,

poi non so compiere - aonore:

ché vertù di tanto savere

sommo chere - laudatore.

Dante non perde l'occasione di occupare quel ruolo e di superare Guittone con la propria lode di Domenico, affidandola alla regia di San Bonaventura.

La ballata di Guittone "Beato Francesco, in te laudare" tratta invece l'elogio del santo di Assisi, e se meno evidenti paiono qui le connessioni col testo dantesco, pure nell'unico manoscritto che ce le tramanda (Laurenziano Rediano 9, ff.5or-5Iv), le due poesie si trovano una dopo l'altra - proprio come si susseguono nel Paradiso dantesco i canti contenenti i panegirici ai due santi - e per di più esse precedono immediatamente la sopracitata ballata "Vegna, vegna - chi vole giocundare", con cui Guittone invita le donne a danzare per il Signore in una manifestazione di ardore sacro che non ha nulla di sensuale, ma tutto dello slancio devozionale che unisce le donne a Cristo e gli uomini alla Chiesa.

Alla luce, dunque, della tradizione lirica religiosa e cortese si può meglio comprendere il fervore religioso che autorizza non solo la performance coreutica dei sapienti e degli apostoli, ma anche l'audace strategia retorica che, ribaltando i generi dei danzanti, sublima la carica erotica del ballo nel fervore mistico delle donne innamorate di Cristo, come i sapienti e i santi sono innamorati della Chiesa. Lo stesso fuoco amoroso che faceva dire al pellegrino in 
attesa di rivedere la sua Beatrice "conosco i segni dell'antica fiamma” ( $P g$. XXX 48), non solo traducendo, ma trasfigurando il senso del verso virgiliano con cui Didone presentiva, nel nuovo amore per Enea, la condanna che l'avrebbe portata al suicidio. La fiamma dell'amore di Dante per Beatrice è simile all'amore degli angeli che danzano intorno a Maria, la "pacifica Orifiamma" ( $P d$. XXXI I27), che fa gli occhi di Dante capaci e "ardenti" (Pd. XXXI I42). Esso è un nuovo tipo di amore, senza eros ma non senza fuoco, così come la danza dei beati e degli apostoli nel Paradiso è un nuovo tipo di arte coreutica, senza dissolutezza ma non senza bellezza e slancio, ma tale altezza di scrittura, con un tale sincretismo di arti diverse, si può raggiungere, per il poeta della Commedia, solo "dall'interno", cioè non ricusando, ma recuperando e trasformando i modelli poetici preesistenti. 


\section{IMMAGINI}
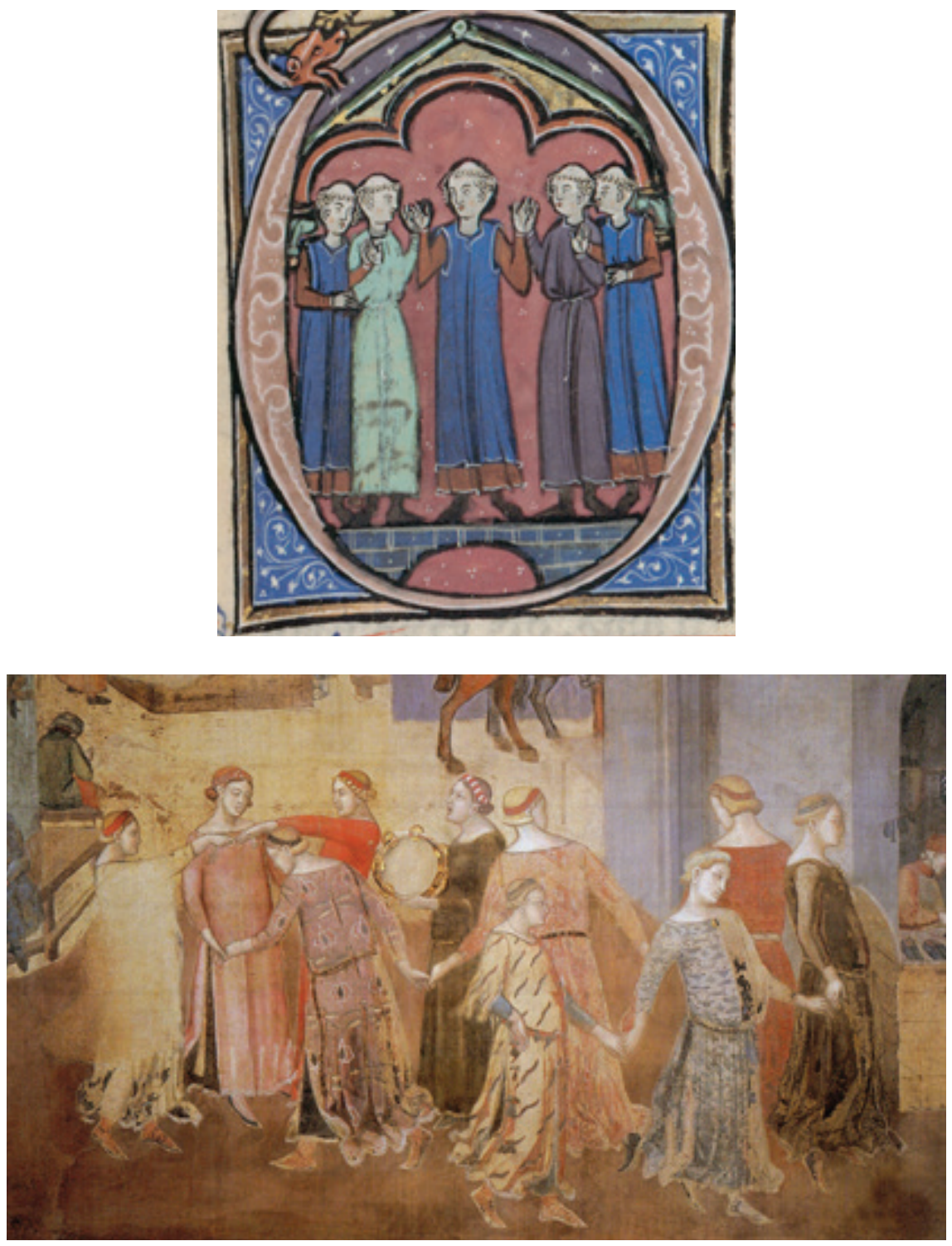

Fig. I. Magnus Liber Organi , f. 428r, Biblioteca Medicea Laurenziana, Plut. 29.I, Firenze.

Fig. 2. Ambrogio Lorenzetti, Allegoria ed Effetti del Buon Governo, I338-1340, dettaglio, Sala della Pace, Palazzo Pubblico di Siena. 

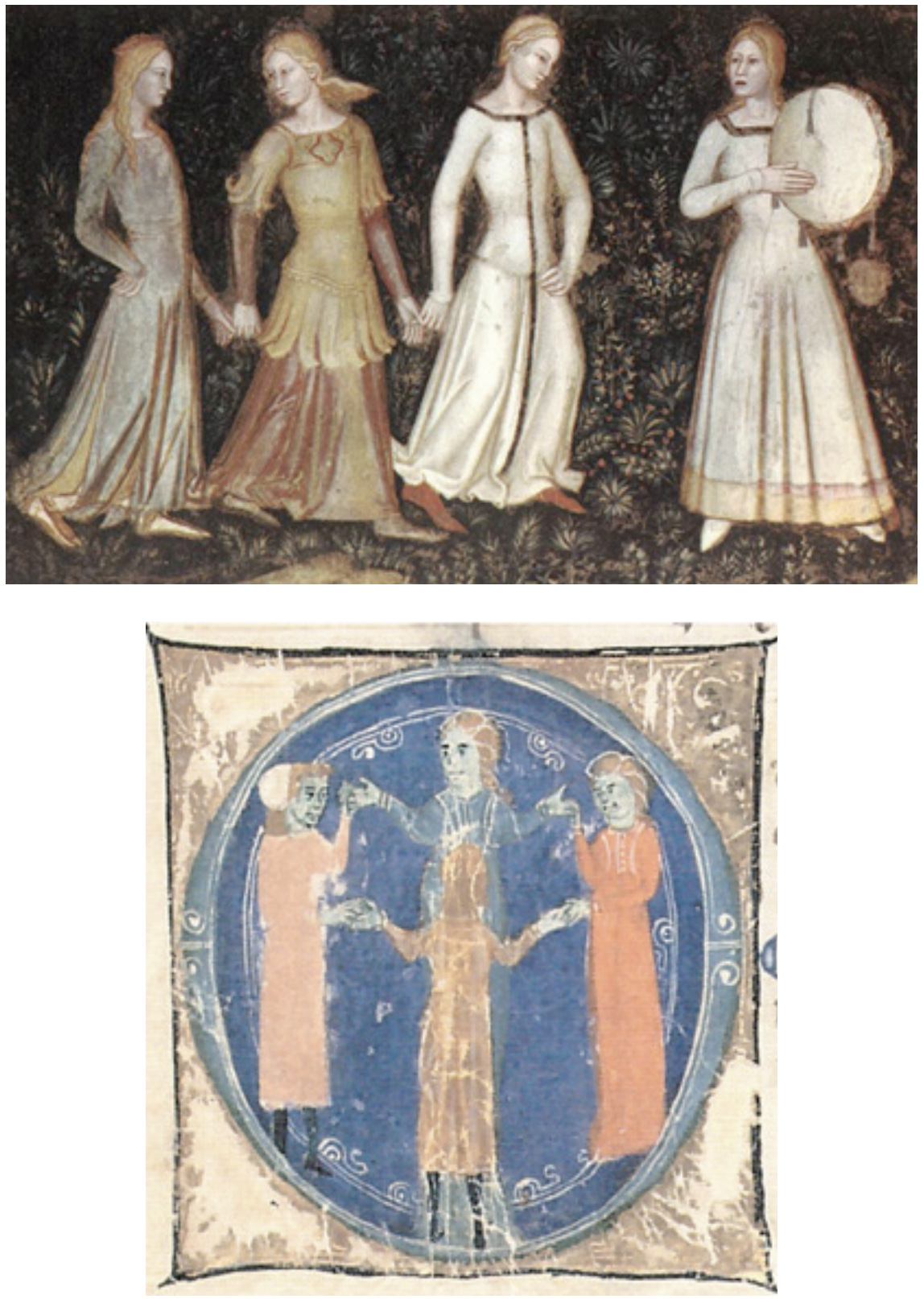

Fig. 3. Andrea di Bonaiuto (Andrea da Firenze), La Chiesa Militante e Trionfante, c. 1365-1367, dettaglio, Cappellone degli Spagnoli, Santa Maria Novella, Firenze.

Fig. 4. Codice Palatino $4 \mathrm{I} 8$ (già Banco Rari 217), f. 58v, Biblioteca Nazionale Centrale, Firenze. 


\section{BIBLIOGRAFIA}

Alberto Magno, I89I, Ethica, a cura di A. Borgnet, J. Echard, J. Quétif, Parigi, Vivèt. Aligheri, D., 1968, La divina commedia, a cura di Natalino Sapegno, Firenze, La Nuova Italia.

Apostolos-Cappadona, D., I993, "Scriptural women who danced", in Dance as Religious Studies, a cura di D. Adams e D. Apostolos-Cappadona, Eugene, Wipf \& Stock.

Anglès, H., 1975, Scripta musicologica, vol. 2, Roma, Edizioni di storia e letteratura.

Ardissino, E., 2009, Tempo liturgico e tempo storico nella Commedia di Dante, Roma, Libreria editrice vaticana.

Barbi, M. e Maggini, F., I956, Rime della "Vita nuova" e della giovinezza, Firenze, Le Monnier.

Barolini, T., 1984, Dante's Poets. Textuality and Truth in the Comedy, Princeton, Princeton University Press.

Bartoli, A., I880, I primi due secoli della letteratura italiana, in Storia letteraria d'Italia scritta da una società d'amici sotto la direzione di Pasquale Villari. vol. 2, Milano, Mallardi.

Blume, C. e Dreves, G., I898, Analectica Hymnica Medii Aevi. Historiae Rhythimicae. Liturgische Reimofficien des Mittelaters. vol. XXVIII, Lipsia, Reisland.

Bonaventura de Bagnorea, I889, Commentaria in Quatuor Libros Sententiarum Magistri Petri Lombardi, Firenze, Quaracchi.

Borghini, V., I897, Bullettino della Società Dantesca, n.s. IV, pp. I8o-8I.

Bosco, U., I970, Enciclopedia dantesca, Roma, Istituto dell'Enciclopedia Italiana.

Buttà, L., 20I4, "Danza en los márgenes: sobre las escenas cortesanas en el Salterio de la reina María", in Danses imaginades, danses relatades paradigmes iconogràfics del ball des de l'antiguitat clàssica fins a l'edat mitjana, a cura di L. Buttà et al., Institut Català d'Arqueologia Clàssica Tarragona, pp. I09-I22.

Canettieri, P. (a cura di), 2009, Iacopone da Todi e la poesia religiosa del Duecento, Milano, BUR.

Cappuccio, C., 2008, "Strutture musicali del cielo del Sole", Tenzone, 9, pp. I47-I78.

Catelli, N., 2008, "Coreografie paradisiache. Le danze dei sapienti (Par. X-XIV)", L'alighieri, 32, pp. II9-I38.

Cavalca, D., I837, Pungilingua di fra Domenico Cavalca, a cura di G. Bottari, Milano, Silvestri.

Concilia Aevi Carolini, 1908, in Monumenta Germaniae Historica inde ab anno Christi quingentesimo usque ad annum millesimum et quingentesimum, Tomus II, Concilia Aevi Karolini I, Pars II, Hannoverae, Impensis Bibliopolii Hahniani. Cirilli, R., 1913, Les Prêtres danseurs de Rome. Étude sur la corporation sacerdotale del Saliens, Parigi, Paul Geuthner.

Contini, G. (a cura di), I960, Poeti del Duecento, Milano-Napoli, Ricciardi.

D’Andria, M., I969, "Inferno, VII, 24", L'Alighieri, I, pp. 5I-57.

Dronke, P., I984, "The Song of Songs and Medieval Love-Lyric", in The Medieval Poet and His World, Roma, Edizioni di storia e letteratura, pp. 209-236

Foster, K. e Boyde, P., 1967, Dante’s Lyric Poetry. Commentary, vol. II, Oxford, Clarendon Press.

Fratini, R., 20I4, "Vacabimus. Appunti per una cinesiologia dantesca", in Danses imaginades, danses relatades paradigmes iconogràfics del ball des de l'antiguitat clàssica fins a l'edat mitjana, a cura di L. Buttà et al., Institut Català d'Arqueologia Clàssica Tarragona, pp. 85-Io8. 
Freccero, J., 1989, "La danza delle stelle: Paradiso X", in La poetica della conversione, Bologna, Il Mulino, pp. 289-317.

Gentile, L., I889, I codici palatini della R. Biblioteca nazionale di Firenze, vol. I, Roma, Fratelli Bencini.

Giordano da Rivalto, I839, Prediche del beato fra Giordano da Rivalto dell'ordine dei predicatori: recitate in Firenze, vol. 3, Milano, Silvestri.

Guérard, B., I850, Cartulaire de l'église Notre-Dame de Paris, vol. 3, Parigi, Crepelet.

Gougaud, L. I9I4, La danse dans les églises, Revue d'Histoire ecclésiastique, no. I5, 5-22, 229-245.

Haines, J. D., 2010, Medieval Song in Romance Languages, Cambridge, Cambridge University Press.

Hollander, R., I996, “Dante's Harmonious Homosexuals (Inferno I6.7-90)”, in Dante Notes http://www.princeton.edu/ - dante/ebdsa/rh.html

Horowitz, J., 1989, "Les danses cléricales dans les églises au moyen age", Le Moyen Age, 95.2, pp. 279-292.

Kay, T., 20I6, Dante's Lyric Redemption. Eros, Salvation, Vernacular Tradition, Oxford, Oxford University Press.

Ledda, G., 20I5, "La danza e il canto dell' "umile salmista»: David nella 'Commedia' di Dante”, in Les figures de David à la Renaissance, a cura di E. Bollet, S. Cavicchioli, P.A. Mellet, Ginevra, Librairie Droz, pp. 225-246.

Lograsso, A. H., I965, "From the Ballata of the 'Vita nuova' to the Carols of the 'Paradiso'", in Dante Studies, 83, pp. 23-48

Martène, E., I7I7, Statuta synodalia Petri Bertrandi episcopi Aedvensis edita, in Thesaurus novus anecdotorum, vol. IV, Lutetiae Parisiorum, Florentinus Delaulne.

Marti, M. (a cura di), 1959, La prosa del Duecento, Milano, Ricciardi.

McGee, T., 1989, Medieval Instrumental Dances, Bloomington, Indiana University Press.

Mingardi, M., I990, "La musica da danza italiana tra XIV e XV secolo: raffronto e considerazioni tra il repertorio strumentale del Codice London B.B. Add. $29987 \mathrm{e}$ le intonazioni dei trattati quattrocenteschi di danza", in Guglielmo ebreo da Pesaro e la Danza nelle corti italiane del XV secolo (Atti del Convegno Internazionale di Studi, Pesaro Villa Miralfiore I6-I8 July 1987), a cura di M. Padovan, Pisa, Pacini, pp. 272-289.

Moleta, V., 1976, The Early Poetry of Guittone d'Arezzo, Londra, The Modern Humanities Research Association.

Mullally, R., 20Io, The Carole: A Study of a Medieval Dance, Burlington, Ashgate.

Nardi, B., I956, "Il canto decimo del Paradiso", Convivium, XXIV, pp. 650-660.

Neville, J., 2008, Dance, Spectacle, and the Body Politick, I250-I750, Bloomington, Indiana University Press.

Padovan, M., 1985, "Da Dante a Leonardo: la danza italiana attraverso le fonti storiche", La danza italiana, 3, pp. 5-37.

Page, C., 1986, Voices and instruments of the Middle Ages: instrumental practice and songs in France II00-I300, Berkeley, University of California Press.

Page, C. 1989, The Owl and the Nightingale: Musical Life and Ideas in France II00-I300, Londra, Dent.

Pasquini, E., 200I, Dante e le figure del vero: la fabbrica della Commedia, Milano, Mondadori.

Pietrini, S., 2000, "La santa danza di David e il ballo peccaminoso di Salomé. Due figure esemplari dell'immaginario biblico medievale", Quaderni Medievali, 50, pp. 45-73. 
Pietrini, S., 20I0, "Danzatrici e cavalle del diavolo: La concezione del ballo femminile nel Medioevo", Viator, 4I, pp. 233-257.

Rokseth, Y., I947, "Danses cléricales du XIII" siècle”, Mélanges 1945. Études historiques, vol. 3, Parigi, Les Belles Lettres, pp. 93-I26.

Rokseth, Yvonne. "Les femmes musiciennes du XIIe au XIVe siècle," Romania 6I Numéro 244 (1935) pp. 464-480.

Sachs, C., 1966, Storia della danza, Milano, Il Saggiatore.

Secor, J. R., I993, "Three dances of three: the imago trinitatis in Dante's Commedia", in Medieval Numerology. A Book of Essays, a cura di R. Surley, New York and London, Garland, pp. 93-IO4.

Skinner, Q., (1999) “Ambrogio Lorenzetti’s Buon Governo Frescoes: Two Old Questions, Two New Answers" in Journal of the Warburg and Courtauld Institutes, Vol. 62 (I999), pp. I-28.

Spanke, H., I930, "Tanzmusik in der Kirche des Mittelalters", Neuphilologische Mitteilungen,31.3/4, pp. I43-I70.

Sparti, B. (2015). Dance, Dancers and Dance-masters in Renaissance and Baroque Italy, Massimiliano Piretti Editore, Bologna.

Stefano di Borbone, 1877, "Diabolus est choreizancium et danciarum inventor", in Anecdotes historiques, légendes et apologues, a cura di A. Lecoy de La Marche, Paris, Renouard, pp. 397-398.

Tani, G., 1983, Storia della danza dalle origini ai nostri giorni, Firenze, Olschki.

Werminghoff, A. (a cura di), I908, Concilium Romanum, canon. XXXV, in Concilia Aevi Carolini, Hanover, Hahn.

Wilson, B., e Barbieri, N., 1995, The Florence Laudario : an edition of Florence, Biblioteca nazionale centrale, Banco rari I8, vol. 29, Madison, A-R Editions.

Vettori, A., I997, “La breccia silenziaria in 'Purgatorio' X, Lectura Dantis, XX-XXI, pp. 78-IOo.

Zimei, F., 20I0, “'Tucti vanno ad una danza per amor del Signore’ Riflessioni pratiche sul rapporto fra lauda e ballata", Studi musicali, n.s. I.2, pp. 313-344. 
